\title{
Construction of Chinese Language Teaching System Model Based on Deep Learning under the Background of Artificial Intelligence
}

\author{
Bochun Kang $\mathbb{D I}^{1}$ and Sicheng Kang ${ }^{2}$ \\ ${ }^{1}$ Department of Language and Literature, Anyang Preschool Education College, Anyang 455000, China \\ ${ }^{2}$ Northeast Agricultural University School of Electrical and Information, Harbin 150000, China \\ Correspondence should be addressed to Bochun Kang; kangbochun@ayyz.edu.cn
}

Received 22 December 2021; Revised 7 January 2022; Accepted 8 January 2022; Published 4 February 2022

Academic Editor: Tongguang Ni

Copyright (c) 2022 Bochun Kang and Sicheng Kang. This is an open access article distributed under the Creative Commons Attribution License, which permits unrestricted use, distribution, and reproduction in any medium, provided the original work is properly cited.

\begin{abstract}
The openness of modern network intelligent education provides a broader learning space for Chinese language learners. People pay more and more attention to network learning, and network intelligent teaching platforms are constantly emerging under this background. Intelligence is one of the most important characteristics of ITS. However, the current network teaching system is far from enough in the intellectualization of teaching content, form, and process. In order to improve the intelligence level of ITS, this paper studies the use of DL network with strong self-learning ability to build ITS. From the point of view of teaching students in accordance with their aptitude and accurately reflecting students' learning state and characteristics, this paper analyzes the influencing factors in students' learning process, puts forward factors such as students' learning style and learning habits into the construction of a student model, and designs the student model. The future trend of ITS is predicted, and it is pointed out that this field with attractive development prospect is worthy of further research and design.
\end{abstract}

\section{Introduction}

In the network environment, Chinese language teaching workers need to reexamine the traditional communication media and teaching mode from the perspective of teaching practice, change ideas and roles, order the disordered oral Chinese teaching resources, and organically integrate modern information means with traditional language teaching means [1]. Teachers should be good at using network resources to design the learning process, and use the network to obtain each student's learning information to regulate the learning process. Artistic Intelligence (AI), computer science, pedagogy, psychology, and behavioral science are all involved in the development of intelligent computer-assisted instruction systems [2]. The ultimate goal of the study is for the computer system to take on the relevant responsibilities of education and teaching, i.e., to endow the computer system with intelligence so that it can partially replace teachers in order to achieve the best teaching
[3]. The openness of modern network education unquestionably expands the learning space available for Chinese language instruction. People are becoming more interested in network learning, and network teaching platforms are continuing to emerge as a result. However, managers and teachers find it difficult to obtain dynamic learning information from network learners due to the increasingly complex particularity and temporal and spatial separation of the network education environment [4]. This results in a simple reproduction of the book content of resources and one-sided pursuit of quantity and scale in the push mode of teaching resources [5]. With a diverse group of online learners and a large number of them, determining how to collect reliable learning status information and provide personalized learning services has become a pressing issue [3].

With the development of multimedia technology and network technology, building a new Chinese language model that breaks the tradition and is not limited by region and 
time is an important issue that needs to be solved urgently [6]. In order to improve teaching efficiency and better train talents, people have been constantly exploring and trying to use new technologies and methods to improve teaching and learning methods and means [7]. At the same time, we hope to teach students according to their aptitude and carry out differentiated education according to students' different learning foundation, learning ability, and other characteristics [8]. However, due to a lack of teacher resources and teaching efficiency requirements, it has been impossible to teach each student according to their aptitude. The Intelligent Teaching System (ITS) proposal makes it possible to accomplish this goal. By creating an open teaching environment, modern education based on the Internet overcomes the time and space constraints of traditional education [9]. It is critical for effectively utilizing the resource advantages of various existing education systems, realizing rational resource allocation, and developing education. It also offers a viable solution to the problem [10]. This paper proposes and establishes an intelligent Chinese language network teaching system model to address the shortcomings of the existing network teaching system.

Intelligent teaching is an important development direction of computer-aided teaching. It is an open interactive teaching formed by using computer to simulate the teaching thinking process of teaching experts and taking students as the center and computer as the medium [11]. Based on modern educational theory, intelligent teaching introduces the latest achievements of AI, psychology, cognitive science, and other disciplines into computer-aided teaching, seeks the mode of learning cognition by studying the characteristics and process of human learning thinking, and enables students to obtain knowledge through personalized adaptive learning, so as to achieve the purpose of real individualized teaching [12]. The research on intelligent computer-assisted instruction system in China started relatively late. The research work started is mainly concentrated in a few universities and research institutions intermittently, most of them are systems for research and demonstration, and few systems have been strictly evaluated [13]. With the rapid development of intelligent computer-assisted instruction system, it will play a positive role in promoting China's education reform. This paper constructs a Chinese language teaching system model based on deep learning (DL). Based on the analysis of the serious defects of adaptability and personalization in the current online learning system, and on the basis of development practice, this paper discusses how to extract parameters such as behavior data and performance information of online learners by using key technologies such as fuzzy evaluation algorithm and Neural Network (NN) in ITS.

\section{Related Work}

According to literature [14], language teaching workers in a networked environment must reexamine traditional communication media and teaching modes from the perspective of teaching practice, change ideas and roles, and realize the ordering of disordered Chinese teaching resources. Reference [15] obtains a framework and design pattern that can quickly construct a college English teaching system with clear structure and reliable performance through research on the modeling of college English teaching system using UML modeling language. The current network teaching platform, according to literature [16], has a wide range of learning contents, and each platform only focuses on cultivating students' abilities in some aspects, rather than cultivating allround abilities of listening, speaking, reading, and writing. Literature [17] seamlessly blends modern information technology with traditional language teaching methods. According to the literature [18], teachers should be skilled at using network resources to design the learning process and obtaining each student's learning information to regulate the learning process. According to the literature [19], the student model is at the heart of ITS individualized teaching and is responsible for the systematic representation of students' knowledge level, cognitive ability, learning motivation, learning style, learning history change, and other data. According to literature [20], these issues in the current Chinese language network teaching platform must be addressed immediately, and it is critical to develop a comprehensive, interactive, personalized, and feedback Chinese language teaching platform. According to literature [21], the main function of a network teaching platform is to display students' characteristics and attitudes and to provide a foundation for the realization of intelligent teaching objectives, teaching contents, and teaching strategies. Literature [22] proposed that educational objectives should include cognitive ability, motor skills, and emotion, in which the objectives of cognitive ability are divided into six levels according to the complexity of intellectual activities. Literature [23] studies the modeling of network college English teaching system based on UML. Through in-depth research, the framework and modeling diagram of online college English teaching system are designed. Literature [5] proposes that the student model is formed according to the interaction and response history between the students and the system, which can be dynamically modified according to students' learning situation, and the system can carry out individualized teaching through the student model. In view of the shortcomings of the existing teaching system, literature [24] proposed an intelligent network teaching system model. Based on previous studies and the current situation of Chinese language network teaching system, this paper constructs a Chinese language teaching system model based on DL. It also classifies students' learning characteristics and provides different teaching strategies and teaching contents according to different characteristics. The model can dynamically establish a personalized learning environment according to the characteristics of students and can truly realize individualized teaching.

\section{Methodology}

3.1. Theoretical Basis of ITS Construction. The concept of DL is derived from the research of Artificial Neural Network. A multilayer perceptron with multiple hidden layers is a $\mathrm{DL}$ structure. DL combines low-level features to form a more abstract high-level representation attribute category or 
feature to discover distributed feature representations of data. The motivation for studying DL is to establish a NN that simulates the human brain's analysis and learning. It mimics the mechanism of the human brain to interpret data, such as images, sounds, and texts. DL is a general term for a type of pattern analysis method. In terms of specific research content, it mainly involves three types of methods: Convolutional Neural Network, self-encoding NN based on multilayer neurons, and deep confidence network [25]. NN has strong learning ability; that is, the main characteristics of training samples can be abstracted through training, so it has strong fault tolerance and memory association ability, can process information in parallel, and therefore has a faster information processing speed. Applying NN to ITS can effectively improve the system's intelligence, adaptability, and response speed.

The combination of computer-aided education and AI, as well as the use of AI in teaching, is referred to as ITS. The intersection of AI, cognitive science, educational theory, and other disciplines produces it. It can be used as an adaptive learning support system to provide individualized instruction, knowledge, and guidance to students with various needs and characteristics. ITS looks for the mode of learning cognition by studying the thinking characteristics and process of human learning. Simultaneously, it aims for personalized teaching; determining students' personal teaching methods and contents based on psychological characteristics, cognitive level, existing knowledge base, cognitive structure, learning habits, learning style, and motivation; and selecting appropriate teaching strategies, in order to provide students with learning contents and progress that are consistent with their learning characteristics. Students develop their abilities as well as their knowledge through personalized and adaptive learning [26]. ITS is a new method and a new way to realize the modernization of teaching means, as it can fully exploit students' enthusiasm, can assist students in developing their intelligence and ability, and is a new method and a new way to help students develop their intelligence and ability. Expert model, student model, teacher model, and intelligent man-machine interface are the four main components of a typical ITS. The basic structure of ITS is shown in Figure 1.

The student model is one of the core components of ITS intelligence, and ITS can use it to implement personalized teaching for learners. A student model is a data structure that depicts the cognitive characteristics of learners. On the one hand, it keeps track of the learner's name, gender, number, and other basic information; on the other hand, it accurately reflects the learner's knowledge level, learning ability, psychological state, and other factors. The model of students changes dynamically in response to interactions between the students and the system, as well as the learning situation, and serves as the foundation for the system's determination of teaching strategies and resources. The expert model is used to represent relevant knowledge in the teaching field, as well as problem-solving knowledge that experts can use to solve related problems [27]. As for the teacher model, its primary responsibility is to choose appropriate teaching materials and present them to students in appropriate formats, while adhering to certain teaching principles. The key to this module's operation is knowing how to organize the teaching content, or how to teach. The Intelligent interface serves as the system's and users' interactive interface, providing intelligent multimedia knowledge input, user information and behavior acquisition, and knowledge output for other modules. Natural language processing, knowledge base maintenance, student model initialization, teacher model adaptive adjustment, and other functions are all part of the ITS intelligent man-machine interface.

Student model is a data structure representing learners' cognitive state, and it is the basis of ITS intelligent teaching. The dynamic structure of the student model shows the formation and relationship of the four components of the ITS in the learning process, as well as their roles in teaching decision. It can be seen that the student model is a dynamic structure, which tracks students' learning activities. By analyzing students' learning behaviors, it records and adjusts the information describing students' personalized characteristics, such as their knowledge structure, learning ability, and learning habits, so as to draw new teaching strategies. Among them, the decision-making mechanism is in the central position, and its functions are maintenance of relevant databases, generation of decisions, resolution of decision conflicts, etc. The dynamic structure of the student model is shown in Figure 2.

At present, there is a lot of research on ITS, including some student models, but these student models generally have some problems such as lack of intelligence. Student model is the most difficult part of ITS design at present, and it is also a hot issue in ITS research. Therefore, constructing a suitable student model is the focus of ITS construction. The model should be able to timely and correctly reflect the essential characteristics and state of students' learning behavior; that is, it reflects students' mastery and understanding of a certain learning content, learning style, habits and learning ability contained in learning behavior, etc. At present, students' models are generally classified into covering model, differential model, deviation model, and cognitive model.

In the covering model, the domain knowledge that students want to learn and its constraint relation are represented as a directed knowledge structure diagram, the learning state of students is regarded as the subgraph of this diagram, and the learning process is regarded as the approximation process of the subgraph to the original diagram. The system based on this model can get the defects of students' knowledge structure according to the comparison between the domain knowledge structure diagram and the students' knowledge state diagram, so as to recommend the contents to be learned to students. The deviation model records the deviations of the students' problem-solving path from the expert path. These deviations describe some deficiencies of students in this knowledge point and can give specific remedial measures according to the types of deviations. The cognitive model reflects the differences in cognitive ability and cognitive structure of each individual learner. By analyzing the cognitive differences of students, we can understand their initial learning ability and 


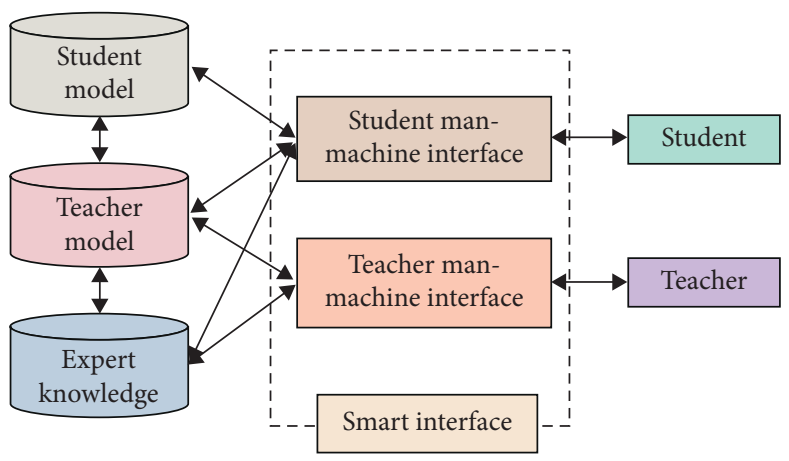

FIGURE 1: Basic structure of ITS.

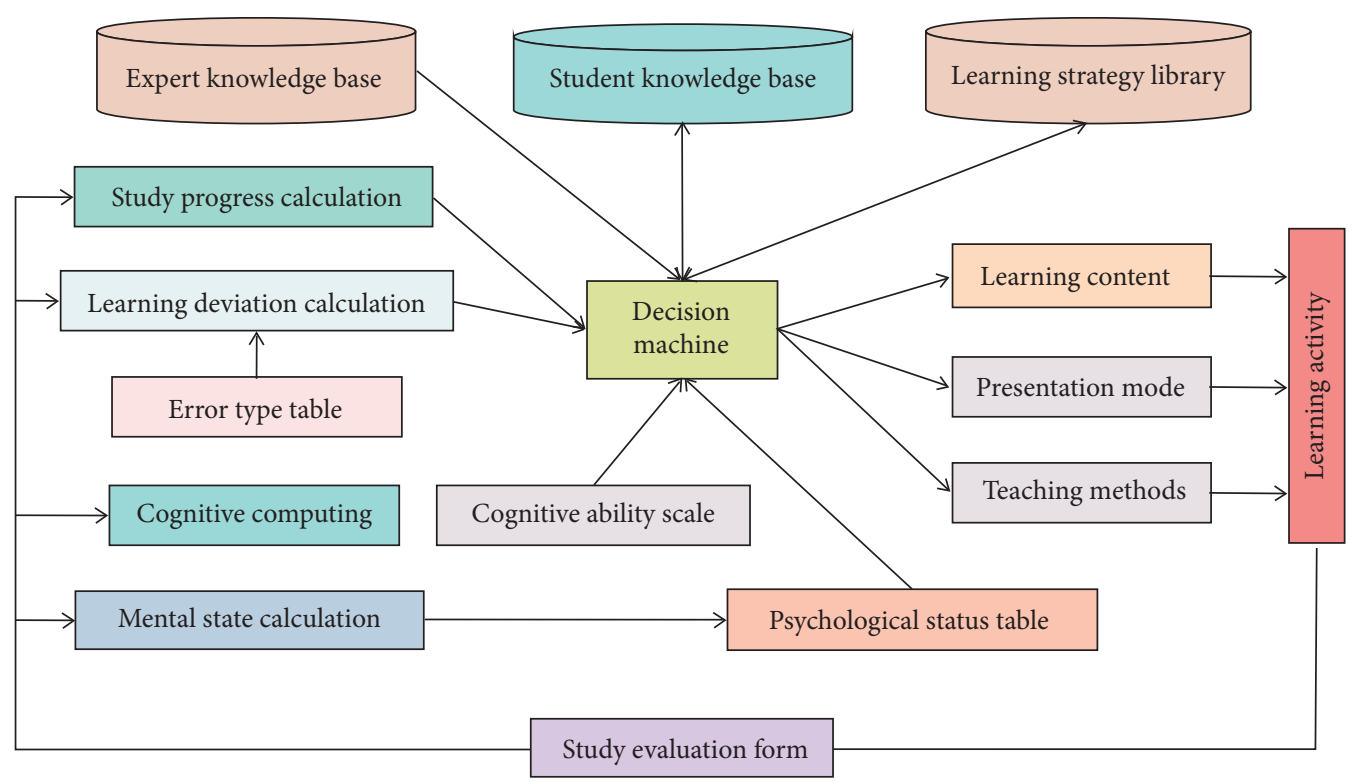

FIgURE 2: Dynamic structure of student model.

knowledge structure. The purpose of analyzing students' cognitive ability and cognitive structure is to formulate effective teaching strategies for specific teaching tasks. The differential model divides learners' knowledge into two parts: the pre-grant and the knowledge beyond the pregrant, which is further expanded than the covering model, but its essence is the covering model.

3.2. Construction of Intelligent Teaching System. Based on the idea of intelligent teaching, the architecture of intelligent network teaching system is designed as three-tier $\mathrm{B} / \mathrm{S}$ structure: user interaction layer, teaching application layer, and database server layer. The user interaction layer includes the interaction interfaces of students, teachers, and system administrators and realizes the interaction between the system and users through browsers. The student interaction interface is the individualized teaching content and learning interface provided by the system for different learners. Students log in to the system first and then participate in the pre-assessment test voluntarily, so that the system can get a preliminary understanding of students' knowledge level, cognitive ability, learning style, favorite learning strategies and so on. In the follow-up study, the system will select the learning contents in the teaching knowledge base that are suitable for students' characteristics and actual level according to the students' learning history, interaction with the system, and performance in the system diagnostic test and dynamically organize students' learning by the teaching strategies in the teaching model. During the learning process, the system dynamically generates the teaching process according to the student model.

Students can choose, judge, and deal with a large amount of knowledge using an intelligent reasoning mechanism in the intelligent teaching assistant system, which makes the learning content more targeted and improves the learning effect. The foundation of all development is system analysis, which is an important stage of software engineering. In the stage of system analysis, an accurate understanding of the system requirements and the internal operating mechanism of the system is helpful in accurately grasping the system requirements, so that the specific content of software development can be correctly determined. To fully understand the users' requirements for the system, we should complete 
the work of determining the content of system requirements, determining all involved elements, and establishing corresponding models for the current problems to be solved through system analysis.

After learning a knowledge point, students first consolidate their knowledge through corresponding exercises and then assess their cognitive ability through tests. The test questions of each knowledge point include three types: single-choice questions, judgment questions, and fill-in-theblank questions. Each question can test at least one of the above six cognitive abilities. When students test, if they answer a question correctly, the cognitive ability of one or more items corresponding to the question will be 1 ; if they answer the question incorrectly, it will be -1 ; and if they do not answer it, it will be 0 . After the students finish the questions of a certain question type, they can calculate each cognitive ability of the students in this question type as follows:

$$
r_{i}=\frac{n_{i j}(1)}{n_{i j}(1)+n_{i j}(-1)}, \quad i=1,2, \ldots 6 .
$$

Among them, $1 \leq j \leq n$, where $n$ is the number of test questions of this question type. $n_{i j}(1)$ is the number of times the $i$-th cognitive ability is answered correctly in this question-type test. $n_{i j}(-1)$ is the number of wrong answers to the $i$-th cognitive ability in this question-type test.

Suppose the test question types for each knowledge point include multiple choice questions, true or false questions, and fill-in-the-blank questions. According to (1), the cognitive abilities of each question type are calculated to form the cognitive ability evaluation of all question types. The matrix is as follows:

$$
R=\left[\begin{array}{l}
r_{11}, r_{12} \ldots r_{16} \\
r_{21}, r_{22} \ldots r_{26} \\
r_{31}, r_{32} \ldots r_{36}
\end{array}\right] .
$$

Among them, $r_{11} \sim r_{16}$ represent the evaluation values of the six cognitive abilities of single-choice questions. $r_{21} \sim r_{26}$ represent the evaluation values of the six cognitive abilities of true and false questions. $r_{31} \sim r_{36}$ represent the evaluation values of the six cognitive abilities of the fill-in-the-blank question.

Knowledge base, as a crucial component of an intelligent teaching assistant system, primarily provides a guiding, adaptive, open, and operable framework, as well as service facilities. It also provides a standardized knowledge input organization for various disciplines' knowledge, as well as other teaching resources that are organized and managed in accordance with it. This will allow users to create their own knowledge system, providing a solid foundation for the development and integration of all types of knowledge applications. In addition, the core knowledge in the teaching field will be decomposed into interrelated knowledge points, forming a knowledge tree, with a visual and operational knowledge tree editing interface that makes it easy for teachers to input teaching knowledge into the knowledge base.
The whole processing process of $\mathrm{NN}$ is a quantitative process for students' participation in a course. The system divides the degree of participation into three levels: positive, general, and negative. The input of $\mathrm{NN}$ includes $n$ variables such as the evaluation value of the three levels of participation, discussion, resources, learning progress, and the frequency of browsing the course web page. The degree of students' participation in the course is selected as the output node, which actually completes a nonlinear mapping from $n$-dimensional space to one-dimensional space.

$$
\begin{aligned}
F_{b p} & : X \longrightarrow Y, \\
X & =\left\{X_{1}, X_{2}, X_{3}, X_{4}, \ldots, X_{n}\right\}, \\
Y & =\{Y\},
\end{aligned}
$$

where $X_{1}, X_{2}, X_{3}$ represent the evaluation values of 3 levels of participation. $X_{4}, \ldots, X n$ express the discussion situation, resource situation, etc. $Y$ represents the degree of student participation in the course.

Learning evaluation table records the learning results of each knowledge point in the unit after each test, which is the basis for adjusting students' knowledge table and error type table, calculating cognitive ability, and then forming teaching strategies. The module of the expert decisionmaking mechanism can be regarded as the inference engine in the intelligent teaching assistant system. Generally, it adopts the method of combining two levels of reasoning, that is, reasoning based on semantic network and reasoning based on production rules. Among them, reasoning based on semantic network is used to determine the teaching content, while reasoning based on production rules is used to determine the teaching strategy. Different from rule-based reasoning, case-based reasoning is regarded as reasoning based on previous experience. Therefore, in some intelligent teaching assistant systems, case-based reasoning is adopted.

The calculation of cognitive ability is to solve the problem of quantitative evaluation and measurement of cognitive ability. According to the different methods of evaluation and measurement of cognitive ability, there can be different methods to determine the cognitive ability of students. Define the weights of various question types: $W=\left(W_{1}, W_{2}, W_{3}\right)$. The weight of a single-choice question is as follows:

$$
W_{1}=\frac{\omega_{1}}{\left(\omega_{1}+\omega_{2}+\omega_{3}\right)} .
$$

The weight of the true or false question is as follows:

$$
W_{2}=\frac{\omega_{2}}{\left(\omega_{1}+\omega_{2}+\omega_{3}\right)} \text {. }
$$

The weight of the fill-in-the-blank question is as follows:

$$
W_{3}=\frac{\omega_{3}}{\left(\omega_{1}+\omega_{2}+\omega_{3}\right)},
$$

$$
W_{1}, W_{2}, W_{3}=1 .
$$

Among them, $\omega_{1}, \omega_{2}, \omega_{3}$ are, respectively, the evaluation value of the average answer of each question type of a 
number of students after the test (the $W_{1}, W_{2}, W_{3}$ value is given by an expert at the beginning and can be dynamically adjusted after a certain number of student tests).

Use (2) and weight $W$ to calculate the final evaluation results of various cognitive abilities:

$$
\begin{aligned}
E & =R \bullet W \\
& =\left(E_{1}, E_{2}, E_{3}, E_{4}, E_{5}, E_{6}\right) .
\end{aligned}
$$

Calculate the comprehensive cognitive ability of students after learning this knowledge point:

$$
S=\sum_{i=1}^{6} E_{i} \times M_{i}
$$

Among them, $M_{i}(i=1,2, \ldots 6)$ is the weight of a certain cognitive ability (the weights of the six cognitive abilities are given by experts). This is a model of the cognitive abilities of students. The learning strategy library keeps track of the learning strategies for each knowledge point acquired through decision in the next round of learning. The next round of learning may consist of relearning a portion of the knowledge from the previous unit or learning a new unit. Determine the learning content, knowledge presentation, teaching methods, and test question generation, among other strategies. In fact, the intelligent interface module is a component that allows the system and users to interact. It not only allows students to input and register their information, but also allows them to communicate with the system. Natural language processing, internal processing of man-machine dialogue, systematic knowledge base maintenance, initialization of student model, adaptive teacher model adjustment, and other technologies are examples of related technologies. This module provides an excellent setting for using negotiation, debate, conversation, and other teaching methods.

The student knowledge base describes and records students' learning progress and level, as well as the types of mistakes made by students in each knowledge point. "Mastery degree" means the students' mastery degree of this knowledge point, and the system can set a threshold. If the knowledge points with test scores higher than the threshold are considered as mastered, the system will no longer arrange learning. Below the threshold, the system will arrange to continue learning. In the vicinity of the threshold, it will prompt students to choose whether to continue to strengthen their knowledge. The "error number" indicates the types of errors made by students in testing this knowledge point. The system can get the corresponding error description information and learning tips from the error type table and provide them to students for targeted learning.

\section{Results Analysis and Discussion}

The cognitive model is an essential component of the student model. People have been studying the human cognitive process for many years. Although some progress has been made, because the human cognitive process is such a complex problem, establishing an ideal student cognitive model at this stage is only an ideal. We must first solve the problem of how to represent cognitive ability in order to determine students' cognitive ability. Students have a variety of preferences. Two preferences are taken into account in this design: knowledge teaching and knowledge presentation. The teaching method of knowledge refers to how students prefer to be educated, and the presentation of knowledge refers to the media in which learning content is presented. We can use graphics to individually represent the data in the model. This is a line chart with coordinates: time, degree of participation, and variable value of cognitive ability, as shown in Figure 3. It has the advantage of being able to intuitively reflect the changing trend of students' related data for teachers to analyze and choose from.

Considering that the student model is mainly used to reflect the dynamic characteristics of students in the learning process, it can provide personalized teaching services for learners. Therefore, we use graphics to represent the data in the model hierarchically. Under the condition of different combinations of participation degree and cognitive ability, the system can automatically choose teaching strategies as a guiding graph. Its advantage is that various teaching resources can be placed in nine areas, analyzed, and selected. The system can provide a brand-new resource service mode of customized service, instant service, and active service for students in different regions, and teachers can also adopt different teaching strategies for students in different regions. The time domain waveform of data distribution obtained by DL algorithm is shown in Figure 4.

It is necessary to consider the cultivation and improvement of students' knowledge memory, classification, induction, and deduction abilities in the ITS student model, as well as reminding students to use deep learning. Thus, the corresponding teacher model can adjust the learning content and teaching methods, while giving suggestions on learning methods, rather than simply letting students learn a certain part of the content again; compare and analyze the questions answered by students; find out the reasons; especially analyze and diagnose the causes of errors; and feed them back to the corresponding teacher model. If the students scored well on the basic knowledge portion of the test but poorly on the comprehensive application test, the students may engage in shallow learning, and the teaching system should provide reminders and suggestions on learning methods, so that the effect of ITS teaching students in accordance with their aptitude can be better reflected. We have experimented on the practicability of the Chinese language intelligent education system, and the distribution of students' scores before and after use is shown in Figure 5.

The experimental results show that most students think that the system can effectively promote students' Chinese language learning, especially their learning cognitive ability. The teaching methods are divided into seven types: focusing on details, detailed description, general description, examples, brief description, prompt, and review. Students can choose a teaching method according to their own characteristics at the beginning of school hours, and the teaching module dynamically adjusts the teaching method during the 


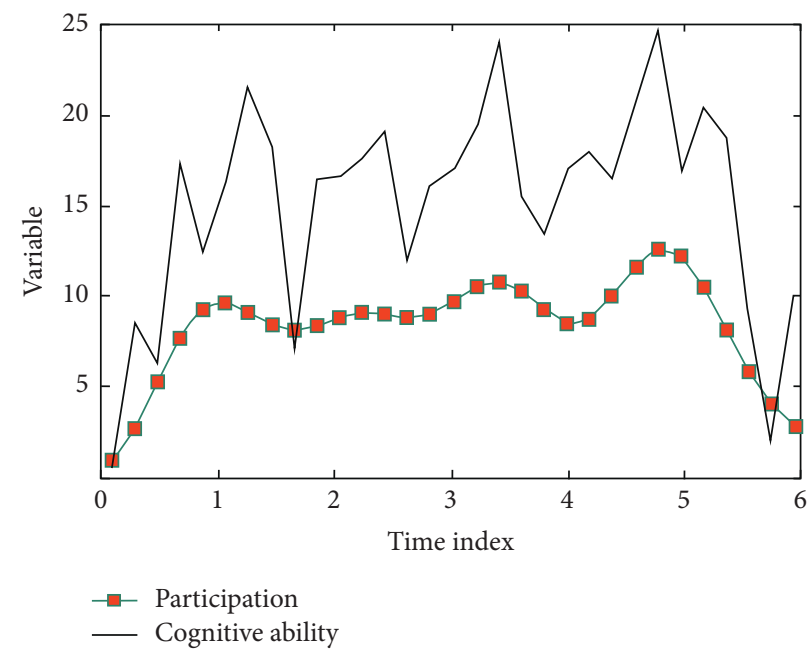

FIgURe 3: Student model line chart.

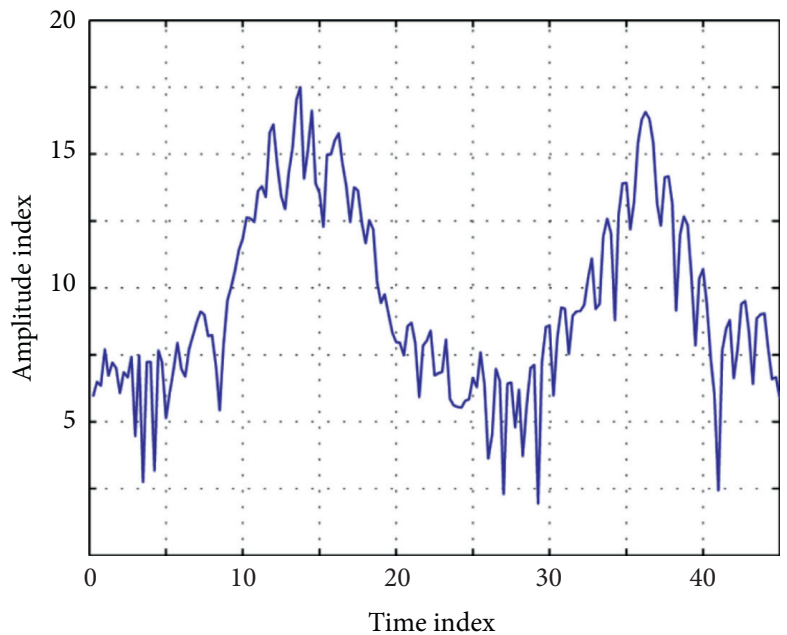

Figure 4: Time domain waveform diagram of data distribution.

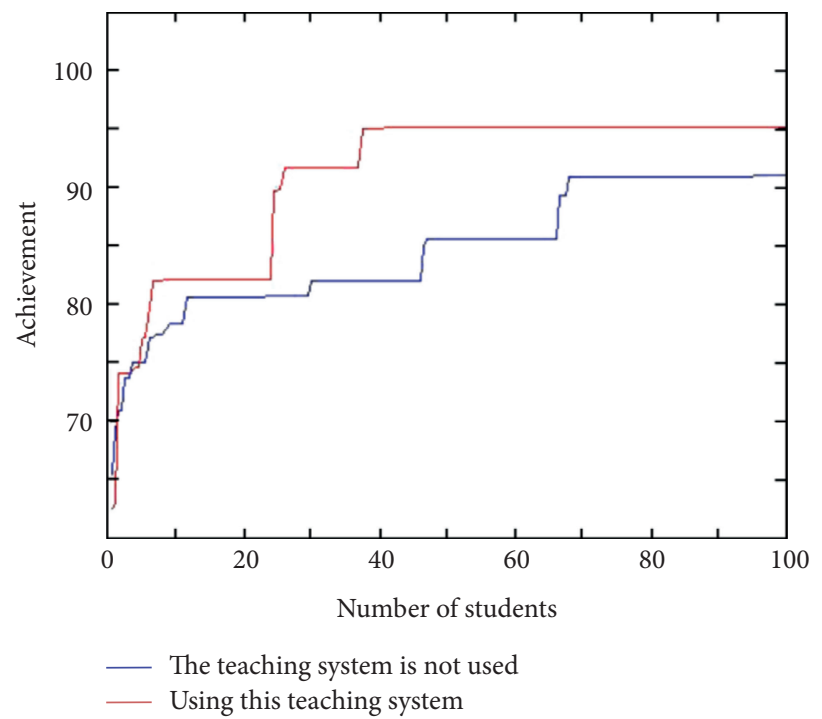

Figure 5: Comparison of student achievement distribution. 
learning process. Teaching strategies are used to adjust the teaching content according to the student model in the teaching process. This strategy library has designed three teaching strategies. (1) Continue to learn this knowledge point. (2) Learn the prerequisite knowledge points for learning this knowledge point. (3) Learn the subsequent knowledge points of this knowledge point. In this experiment, 100 students were selected to use and score the original teaching system and this teaching system. The score data of the original teaching system and this teaching system are shown in Figure 6.

Students' study habits, their age, the correct knowledge they have and the degree to which they have mastered it, their study history, and their personality characteristics, among other things, should all be taken into account when designing the student model. Students' learning behavior in the student model includes total time, time spent learning a specific content, and time spent solving a specific problem. The accuracy rate drops to varying degrees, accompanied by a slight fluctuation, and the classification effect decreases as the feature dimension of Chinese language education increases. The relationship between the dimension of deep learning features obtained from two groups of experiments on the same batch of samples and the discrimination results is shown in Figure 7.

In the process of learning, students may be dissatisfied with or afraid of a certain course because they always make mistakes in doing the questions, thus taking a negative attitude to do or not do it. The system will repeat it according to students' performance until they learn, and students will become more and more bored, which makes the teaching effect not good. After adding students' emotions, the system can help students overcome this psychological state by appropriate means when students are in a bad state. Experiments are carried out on the data obtained in this paper, and the change trend of the target value with the number of iterations is shown in Figure 8.

$\mathrm{NN}$ modifies the connection weights of the network structure through adaptive algorithm, so that the network approaches the expected input-output relationship, can automatically adjust the parameters according to the changes of input data, and can optimize the system to better reflect the learning characteristics of students. The number of input level nodes is 5. The output layer is designed according to the students' learning state and characteristics to be expressed. At present, the set output includes the mastery of a certain learning content, learning methods, and learning habits. The number of hidden layers is set as needed. The model realizes the interaction between the users and the system, and it is the interface of two-way activities of teaching and learning, including student interaction interface, teacher interaction interface, and administrator interaction interface; at the same time, it includes the design of interfaces among the models. The model mainly uses web to express and communicate the content. Because of the

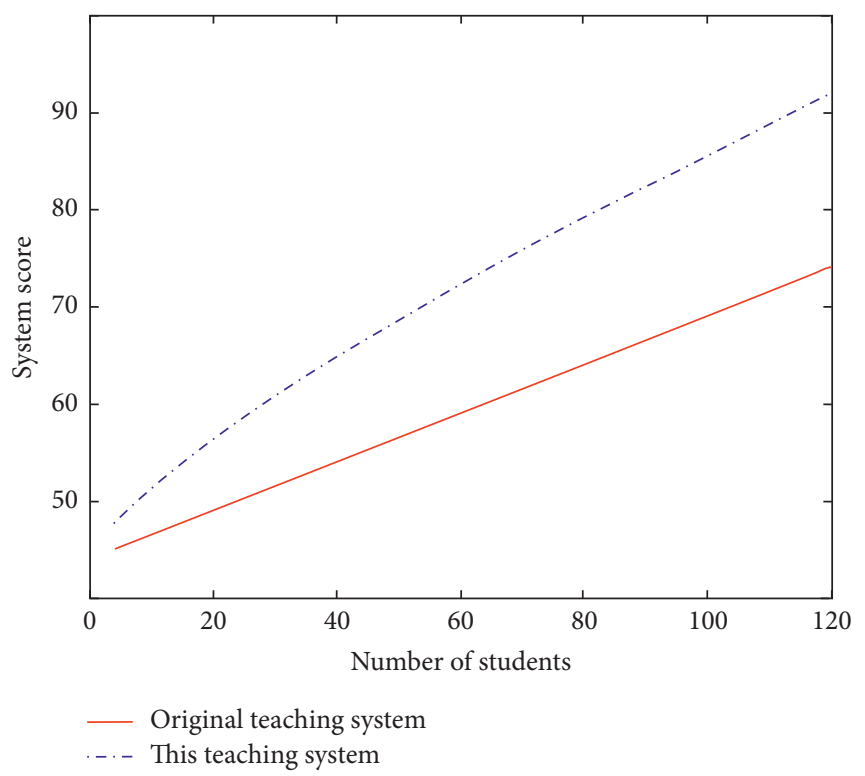

FIgURE 6: The use score of the original teaching system and this teaching system.

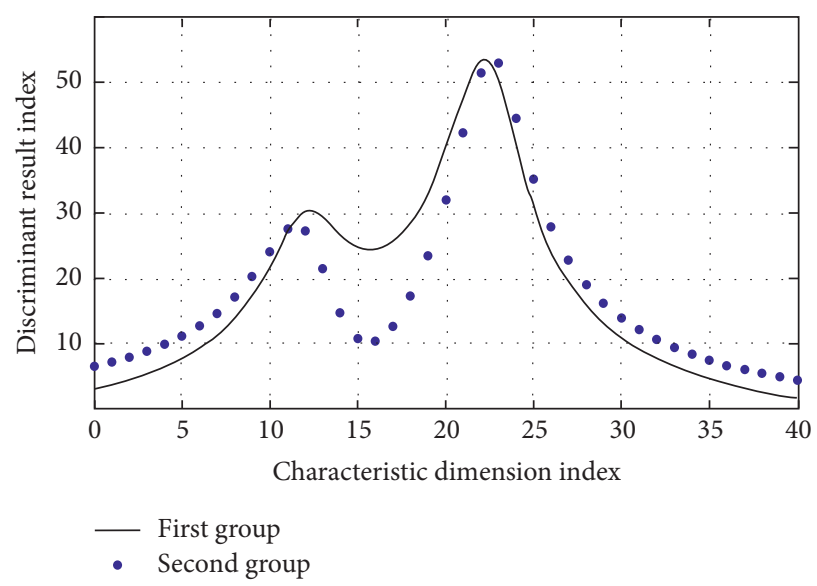

FIgURE 7: Characteristics change of Chinese language education based on DL.

untimely feedback of online learners' learning information, which is common in Chinese language teaching systems, it is difficult for teachers and managers to actively acquire the learning status of online learners. The intelligent network teaching system based on DL is designed and implemented in this section. The system uses NN and other technologies to analyze, mine, and establish student models in a shared environment of network "teaching and learning" with curriculum as the focal point. The basic teaching strategies used in the teaching process are determined based on the individual needs of students, and they are provided with a brand-new resource service mode of customized service, instant service, and active service. 


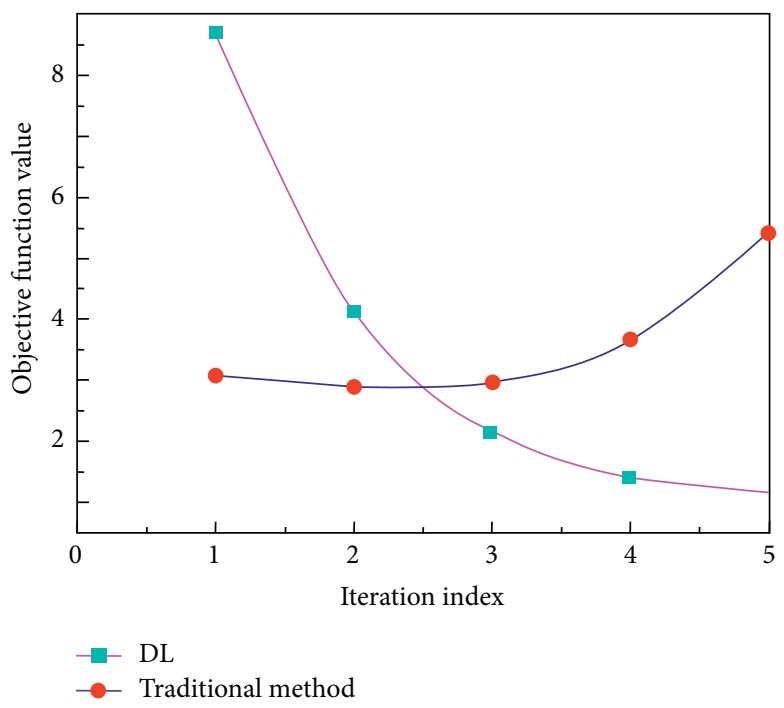

Figure 8: Trend of target value with iteration times.

\section{Conclusions}

In the information age, all things, environment, and information that can be used to achieve the goal of education and teaching can be regarded as educational resources. Therefore, in the process of Chinese language teaching, those that can be used to create Chinese language learning environment can be regarded as teaching resources. In today's educational reform environment, the intelligent teaching assistant system will play an increasingly important role. At present, there are not many achievements in this field in China, and even fewer systems can really be put into teaching practice. This field with attractive development prospects deserves our further study and discussion. Because the learning process and the factors affecting learning are very complicated, it is necessary to further study the factors affecting learning; the description, recording, and evaluation methods of learning activities; and the complex relationship between learning objectives, students' characteristics, teaching strategies, and learning effects and build a more scientific student model. In this paper, object-oriented modeling, teacher model, student model, and background support model are created in the system. It has solved the problems that the current development system is not fully functional and cannot realize personalized and interactive Chinese language teaching. The Chinese language teaching system model based on DL designed in this paper can adjust itself according to students' learning behavior; judge students' learning level, ability, learning style, and other student status and characteristics; and provide necessary information for teaching modules and expert modules, so that it can provide personalized learning resources and environment for students. At the same time, it is of great practical significance to improve the intelligence of ITS and teach students in accordance with their aptitude.

\section{Data Availability}

The data used to support the findings of this study are included within the article.

\section{Conflicts of Interest}

The authors declare that there are no conflicts of interest.

\section{References}

[1] Y. Guo, R. Yan, and J. Wang, "Artificial intelligence meets Chinese medicine," Chinese Journal of Integrative Medicine, vol. 25, no. 9, pp. 10-15, 2019.

[2] S. Graham, C. Depp, E. E. Lee et al., "Artificial intelligence for mental health and mental illnesses: an overview," Current Psychiatry Reports, vol. 21, no. 11, p. 116, 2019.

[3] Z. Zhu, J. Shan, and H. Yan, "International investigation and development strategy for artificial intelligence maker education," Open Education Research, vol. 25, no. 1, pp. 47-54, 2019.

[4] X. Zhang, X. Cao, S. Geng, and T. Tian, "Research on military aided decision intelligence based on deep learning," Journal of Sichuan Armamentary Engineering, vol. 39, no. 10, pp. 162-167, 2018.

[5] D. Pan, G. Qin, and W. Chen, "Application progress of artificial intelligence technology based on deep learning in breast cancer screening and imaging diagnosis," International Journal of Medical Radiology, vol. 42, no. 1, pp. 12-15, 2019.

[6] K. W. Johnson, J. Torres Soto, B. S. Glicksberg et al., "Artificial intelligence in cardiology," Journal of the American College of Cardiology, vol. 71, no. 23, pp. 2668-2679, 2018.

[7] Y. Lv, W. Li, and H. Liang, "On the application of deep learning in artificial intelligence courses," Educational Research, vol. 3, no. 10, pp. 105-106, 2020.

[8] L. Hang, "Deep learning for natural language processing: advantages and challenges," National Science Review, vol. 5, no. 1, pp. 24-26, 2018.

[9] S. Miao, T. Xu, Y. Wu et al., "Extraction of BI-RADS findings from breast ultrasound reports in Chinese using deep learning approaches," International Journal of Medical Informatics, vol. 119, no. 11, pp. 17-21, 2018.

[10] M. Agarwal, L. Saba, S. K. Gupta et al., "A novel block imaging technique using nine artificial intelligence models for COVID-19 disease classification, characterization and severity measurement in lung computed tomography scans on 
an Italian cohort," Journal of Medical Systems, vol. 45, no. 3, pp. 1-30, 2021.

[11] X. Zhang, L. Tan, Y. Wu, and J. Zi, "Case-based teaching and practice of artificial intelligence courses for medical undergraduates," Chongqing Medicine, vol. 49, no. 13, p. 4, 2020.

[12] A. Hirschmann, J. Cyriac, B. Stieltjes, T. Kober, J. Richiardi, and P. Omoumi, "Artificial intelligence in musculoskeletal imaging: review of current literature, challenges, and trends," Seminars in Musculoskeletal Radiology, vol. 23, no. 3, pp. 304-311, 2019.

[13] X. Jiang, T. Jiang, and X. Xuan, “Application of deep learning artificial intelligence technology in medical imaging assisted analysis," China Medical Equipment, vol. 36, no. 6, p. 8, 2021.

[14] S. Liu and Y. Xiao, "Challenges and opportunities of artificial intelligence based on deep learning to medical imaging," Chinese Journal of Radiology, vol. 51, no. 12, p. 3, 2017.

[15] T. Yi, C. Lin, E. Jiang, and Y. Zhong, "Application and prospect of hyperspectral imaging technology fusion deep learning method in the field of traditional Chinese medicine under the vision of artificial intelligence and industry 4.0," Chinese Journal of Chinese Materia Medica, vol. 45, no. 22, p. 5, 2020.

[16] T. Wei, C. Lawrence, and M. D. Abramoff, "Observations and lessons learned from the artificial intelligence studies for diabetic retinopathy screening," JAMA Ophthalmology, vol. 137, no. 9, pp. 994-995, 2019.

[17] Z. Zhang and S. Liu, "Artificial intelligence will surely lead to changes in ideological and political theory courses," Ideological Education Research, vol. 10, no. 10, p. 6, 2020.

[18] J. Qiu, "Research and development of artificial intelligence in China," National Science Review, vol. 3, no. 4, pp. 538-541, 2016.

[19] A. Xu, Z. Zhang, Q. Sun, and H. Tan, "Construction of artificial intelligence air quality forecasting system based on deep learning," China Environmental Monitoring, vol. 37, no. 2, p. 7, 2021.

[20] X. Yang and Z. Zhang, "The progress of artificial intelligence based on deep learning in digital pathology," Chinese Journal of Cancer, vol. 31, no. 2, p. 5, 2021.

[21] L. Zhang and S. Guan, "Learning for understanding: knowledge learning in the era of artificial intelligence," Journal of Educational Science of Hunan Normal University, vol. 20, no. 1, p. 6, 2021.

[22] S. Lin, Y. Qu, L. Chang, L. Yu, and L. Cao, "A review of the development of artificial intelligence in Chinese medicine and discussion on the trend of technology integration," Chinese Journal of Traditional Chinese Medicine, vol. 35, no. 11, p. 6, 2020.

[23] X. Li, "The application value of artificial intelligence based on deep learning in rib fracture detection," Journal of Practical Radiology, vol. 36, no. 11, p. 4, 2020.

[24] Y. Wang, S. Yu, D. Chen et al., "Artificial intelligence design decision model based on deep learning," Computer Integrated Manufacturing Systems, vol. 25, no. 10, p. 9, 2019.

[25] R. Wang, S. Luo, Z. Wu et al., "Application and development trend of deep learning in Chinese semantic analysis," Computer Technology and Development, vol. 29, no. 9, p. 7, 2019.

[26] Y. Wang, F. Yang, J. Zhang, H. Wang, X. Yue, and S. Liu, "Application of artificial intelligence based on deep learning in breast cancer screening and imaging diagnosis," Neural Computing \& Applications, vol. 33, no. 15, pp. 9637-9647, 2021.
[27] Q. Jian, H.-Y. Chen, W. Dai, Q. Lv, and Y. Chen, "Artificial intelligence approach to find lead compounds for treating tumors," The journal of physical chemistry letters, vol. 10, no. 15, pp. 4382-4400, 2019. 